\title{
Verbal processing in patients with bipolar disorder during a manic episode
}

\author{
Vasilis P Bozikas*1, Mary H Kosmidis², Maria Giannakou' ${ }^{2,3}$, \\ Katerina Stafyla ${ }^{3}$, George Garyfallos ${ }^{3}$ and Kostas Fokas ${ }^{3}$
}

Address: ${ }^{1} 1$ st Department of Psychiatry, Medical School, Aristotle University of Thessaloniki, Greece, ${ }^{2}$ Neuropsychology Group, School of Psychology, Aristotle University of Thessaloniki, Greece and ${ }^{32}$ nd Department of Psychiatry, Medical School, Aristotle University of Thessaloniki, Greece

* Corresponding author

from International Society on Brain and Behaviour: 3rd International Congress on Brain and Behaviour

Thessaloniki, Greece. 28 November - 2 December 2007

Published: 17 April 2008

Annals of General Psychiatry 2008, 7(SuppI I):SI54 doi:I0.I 186/I744-859X-7-SI-SI54

This abstract is available from: http://www.annals-general-psychiatry.com/content/7/SI/SI54

(c) 2008 Bozikas et al.; licensee BioMed Central Ltd.

\section{Background}

Dichotic listening tests have been used to study cerebral lateralization for language and thus the functional integrity of the left temporal lobe. The aim of the present study was to investigate dichotic listening performance, as well as the impact of acute symptom improvement on performance, in a group of Greek patients with bipolar disorder during a manic episode.

\section{Materials and methods}

Participants were 20 patients with a DSM-IV diagnosis of bipolar disorder during a manic episode and 22 healthy controls matched on age, education and gender ratio. Participants were assessed with a computerized dichotic listening task presenting fused simple words, a presumed measure of lateralized temporal lobe language processing. The examinees were instructed to repeat the words they had just heard without any other specific instruction. The variable of interest was the total number of the words reported correctly separately for each ear; when both words were repeated, only the first one was counted as correct. Both groups were evaluated twice within an interval of approximately 4 weeks; patients underwent clinical and neuropsychological assessment at the beginning and again at the end of their hospitalization (mean duration: 26.5, SD: 11 days).

\section{Results}

Repeated measures analyses of variance revealed a significant main effect only for Group $[\mathrm{F}(1,40)=42,81$, p < $0.001]$, with the manic patients reporting fewer correct words than the healthy comparison group, but neither for Ear $[F(1,40)=0.31, p=0.58]$ nor for Time of testing $[F(1,68)=2.45, p=0.13]$. We found no significant interaction between Group x Ear $[\mathrm{F}(1,40)=2.56, \mathrm{p}=0.18]$, Group $\mathrm{x}$ Time $[\mathrm{F}(1,40)=0.35, \mathrm{p}=0.56]$, or Group $\mathrm{x}$ Ear $\mathrm{x}$ Time $[\mathrm{F}(1,40)=1.59, \mathrm{p}=0.21]$.

\section{Conclusions}

Patients with bipolar disorder during a manic exacerbation showed an attentional deficit, which remained consistent even after substantial symptom improvement. 